\title{
Retinal Nerve Fiber Layer and Future Risk of Multiple Sclerosis
}

\author{
Fiona Costello, William Hodge, Y. Irene Pan, Luanne Metz, Randy H. Kardon
}

\begin{abstract}
Background: Optical coherence tomography (OCT) - measured retinal nerve fiber layer (RNFL) values may represent a surrogate biomarker for axonal integrity in multiple sclerosis (MS). The purpose of this study was to determine whether RNFL measurements obtained within two years of an optic neuritis (ON) event distinguish patients at increased risk of developing clinicallydefinite MS (CDMS). Methods: Fifty consecutively sampled patients who experienced a single ON event were followed prospectively for a mean period of 34 months with OCT testing. Values of RNFL in clinically-affected and non-affected eyes were compared between patients who developed CDMS and those that did not develop MS after ON. Findings: Twenty-one patients (42\%) developed CDMS during the course of the study, with a mean conversion time of 27 months. Mean RNFL values were thinner in the clinically - affected eyes of non - MS patients than CDMS eyes after one year $(p=0.0462)$ due to more severe ON events in the former. By year two, CDMS patients manifested more recurrent ON events and RNFL thinning than non - MS patients. Temporal RNFL values were thinner in the non-affected eyes of CDMS patients with a trend towards significance $(p=0.1302)$. Interpretation: Our results indicate that RNFL thickness does not reliably distinguish patients at higher risk of converting to CDMS after ON. The severity of ON has a greater effect on RNFL thickness than risk of CDMS at one year. The CDMS patients demonstrate progressive RNFL thinning likely due to recurrent sub-clinical ON events, which may help differentiate them from non - MS patients over time.
\end{abstract}

RÉSUMÉ: Couche de fibres nerveuses de la rétine et risque futur de sclérose en plaques. Contexte : La mesure de la couche de fibres nerveuses rétiniennes (CFNR) par tomographie en cohérence optique (TCO) pourrait constituer un biomarqueur-substitut de l'intégrité axonale dans la sclérose en plaques (SEP). Le but de cette étude était de déterminer si les mesures de la CFNR obtenues dans les deux années qui suivent un épisode de névrite optique (NO) peuvent identifier les patients à risque plus élevé de présenter une SEP certaine au point de vue clinique (SEPCC). Méthodes : Cinquante patients consécutifs ayant présenté un seul épisode de NO ont été suivis de façon prospective pendant en moyenne 34 mois au moyen de la TCO. Les mesures de la CFNR de l'œil atteint et de l'œil non atteint ont été comparées entre les patients qui ont éventuellement présenté une SEPCC et ceux qui n'en ont pas présenté après la NO. Résultats : Vingt et un patients (42\%) ont présenté une SEPCC pendant la durée de l'étude. Le délai d'apparition moyen de la SEP était de 27 mois. Après un an, les mesures moyennes de la CFNR étaient plus basses dans les yeux atteints des patients sans SEP que dans les yeux des patients qui ont éventuellement présenté une SEPCC $(p=0,0462)$ à cause d'une NO plus sévère chez les patients sans SEP. La deuxième année, les patients atteints de SEPCC présentaient plus d'épisodes de NO récurrente et d'amincissement de la CFNR que les patients sans SEP. Les mesures de la CFNR temporale avaient tendance à être plus basses dans l'œil non atteint des patients présentant une SEPCC, sans atteindre le seuil de la signification statistique $(\mathrm{p}=0,1302)$. Interprétation : Nos résultats montrent que l'épaisseur de la CFNR n'identifie pas de façon fiable les patients à plus haut risque de présenter une SEPCC après une NO. La sévérité de la NO avait un effet plus important sur l'épaisseur de la CFNR que le risque de SEPCC un an plus tard. Les patients atteints de SEPCC présentent un amincissement progressif de la CFNR qui est probablement dû à des épisodes sub-cliniques récurrents de NO, ce qui pourrait avec le temps aider à les distinguer des patients non atteints de SEP.

Can. J. Neurol. Sci. 2008; 35: 482-487

Optic neuritis (ON) is an inflammatory injury of the optic nerve, which represents with first demyelinating event in approximately $20 \%$ of patients with multiple sclerosis (MS). ${ }^{1,2}$ The Optic Neuritis Treatment Trial ${ }^{3}$ showed that risk of future clinically definite MS (CDMS) is increased among ON patients with white matter lesions on their baseline magnetic resonance imaging (MRI) study. ${ }^{4,5}$ Conversion to CDMS is decreased among ON patients with no baseline MRI lesions and the following clinical features at presentation: male gender, absence of pain, no light perception vision, marked optic disc edema, peri-papillary hemorrhages and retinal exudates. ${ }^{5}$ The approach to MS management has become increasingly proactive in recent years. Clinical trials have demonstrated that disease-modifying therapies can diminish the rate of conversion to CDMS in

From the Hotchkiss Brain Institute, University of Calgary, Departments of Clinical Neurosciences (FC, LM) and Ophthalmology (FC), Calgary, Alberta; The University of Ottawa (WH, YIP), Department of Ophthalmology, Ottawa, Ontario, Canada; The University of Iowa (RHK) and Veterans Administration Hospital, Department of Ophthalmology and Visual Sciences, UIHC, Iowa City, Iowa, USA.

Received February 21, 2008. Final Revisions Submitted April 9, 2008. Correspondence to: Fiona Costello, 5th Floor, Holy Cross Ambulatory Care Centre, Rockyview Hospital, 7007 - 14th Street SW, Calgary, Alberta, T2V 1P9, Canada. 
patients with $\mathrm{ON}$ and other clinically-isolated syndromes, if initiated as early as the first demyelinating event. ${ }^{6-8}$ Accordingly, efforts are ongoing to identify new clinical tools, which capture the effects of sub-clinical disease activity and distinguish patients at increased risk of MS-related disability.

One technology, which has generated recent interest in MS research is a non-invasive ocular imaging device called optical coherence tomography (OCT).$^{9-19}$ Optical coherence tomography uses low-coherence interferometry to generate high resolution $[\leq 10$ microns $(\mu \mathrm{m})]$, cross-sectional images of the retinal nerve fiber layer (RNFL) by measuring backscatter of infrared light. Optical coherence tomography imaging is analogous to ultrasound, except that the time-of-flight delay is measured for light instead of sound. ${ }^{20-23}$ The RNFL contains the retinal ganglion cell axons that comprise the optic nerve; and because it lacks myelin, represents a unique region of the central nervous system. Retinal nerve fiber layer defects are caused by retrograde degeneration of axons damaged by optic nerve inflammation in ON and MS patients. ${ }^{10,24}$ The OCT-measured RNFL values are diminished among ON and MS patients.9-19 Furthermore, reduced RNFL values in ON and MS patients have been shown to correlate with: diminished visual and neurological function, ${ }^{9-13,16,18,19}$ reduced optic nerve magnetization transfer ratios, ${ }^{15}$ MRI-measured optic nerve and brain atrophy, ${ }^{14,17}$ and decreased cerebral brain matter volumes. ${ }^{16}$ In MS patients, OCTmeasured RNFL values have also been correlated with disease progression and relapses. ${ }^{11,16,18,19}$

The evidence supporting OCT as a structural biomarker of optic nerve integrity in $\mathrm{ON}$ and MS patients continues to mount. Yet, the value of OCT-measured RNFL values in capturing subclinical disease activity, and thus identifying clinically isolated syndrome (CIS) patients at risk for CDMS after ON is not known, because prospective data are lacking. In this study, we compared RNFL values between ON patients who developed $\mathrm{CDMS}^{25}$ to patients who did not develop MS (non - MS patients) after ON. Our primary objective was to determine whether RNFL values could be used to distinguish patients at greater risk for CDMS after ON. We aimed to use this data to model expected RNFL changes and events over the two-years that follow $\mathrm{ON}$ in CIS patients, in order to design future intervention studies.

\section{METHODS}

Study design and Sampling. This was a prospective cohort of consecutively sampled patients assessed in the NeuroOphthalmology Clinics at the Ottawa Hospital (FC) between January 2003 and June 2007. The study received approval from the ethics board at the Ottawa Hospital, and participating patients provided informed and written consent.

Inclusion and Exclusion Criteria. Fifty consecutive patients (100 eyes) who experienced a single clinical unilateral ON event were included in the study. Patients were diagnosed with $\mathrm{ON}$ if they demonstrated the following clinical features: decreased visual acuity, a visual field defect which followed the topography of the retinal nerve fiber layer, color vision loss, a relative afferent pupil defect, and a compatible fundus examination (mild or no optic disc edema, and the absence of pallor at the time of the acute event). Exclusion criteria included other established causes of vision loss in the affected eye (including amblyopia, glaucoma, and dense cataracts), a known diagnosis of MS, and inability to undergo reliable OCT testing.
Outcome measures. The primary outcome measure in this study was the comparison of RNFL values in clinically-affected eyes and non-affected eyes between patients who converted to CDMS (CDMS patients) and patients who did not develop CDMS (non - MS patients) after ON. For the purposes of this study, CDMS was defined according to Poser criteria ${ }^{25}$ and included patients who experienced two clinical attacks, separated by a period of at least one month, with documented neurological signs of lesions in more than one area. Visual function scores after ON were compared between CDMS and non - MS patients, because differences in visual recovery could affect RNFL comparisons between the two groups.

Other Variables. Demographic and clinical variables including age; gender; the presence of pain; lesion burden on the baseline MRI scan; mono-focal ON (ON without other neurological symptoms) versus multi-focal ON (ON plus neurological symptoms referable to a region of the central nervous system different from the afferent visual pathway) presentation; treatment with high dose corticosteroids; and the initiation of disease-modifying agents (Interferon beta - 1a, interferon beta - $1 \mathrm{~b}$, or glatiramer acetate) were recorded. The time to baseline MRI acquisition and MRI protocols varied between patients. For this reason, we were not able to correlate specific MRI parameters with RNFL values in this study; nor, were we able to employ radiological criteria to define conversion to MS after ON. ${ }^{26}$ Instead, we documented whether patients had white matter lesions ${ }^{6}$ or a normal MRI scan at the time of the ON event. The treating physician employed individualized discretion concerning the decision to administer corticosteroid therapy for acute ON. Patients who received corticosteroid therapy were treated within two weeks of the ON event with the equivalent of $1000 \mathrm{mg}$ intravenous methly-prednisolone daily for three days. Disease-modifying drugs were administered to a minority (24\%) of patients in this study. No patients initiated disease-modifying agents earlier than six-months after ON. The limited number $(12 / 50)$ of patients who received these therapies precluded efforts to compare the effects of disease-modifying drugs on RNFL values after ON.

Clinical Assessment. Patients underwent repeat neuroophthalmic assessment and OCT testing at approximately threesix month intervals for a minimum period of 24 months. The RNFL values between CDMS and non - MS patients were compared at year one and year two after ON. Neuro-Ophthalmic Assessment: With each visit, patients were questioned regarding new and residual subjective symptoms of vision loss, and underwent a complete neuro-ophthalmic evaluation (FC). The assessment included best-corrected Snellen visual acuity (converted to $\log$ MAR [log (minimal scale of resolution) ${ }^{27}$ visual field analysis, and dilated ophthalmoscopy. Visual field mean deviation (VFMD) measured in decibels (dB)] was determined with Humphrey perimetry 30-2 full threshold SITA algorithm (Zeiss Meditech-Dublin, California). Humphrey test results were used if the false positive, false negative and fixation loss scores measured less than one third. Neurological testing: Patients underwent clinical evaluations at six-month intervals by a neurologist in the MS Clinic at the Ottawa Hospital. Patients aged greater than 45 years, or those with atypical features (including incomplete recovery after $\mathrm{ON}$ ) underwent visual electro-diagnostic testing (including pattern visual evoked potential, and multi-focal electroretinogram studies) to exclude 
retinal mimics of ON. If clinically indicated, additional studies were also performed to exclude conditions that could mimic MS such as myeloproloferative disorders, syphilis, sarcoidosis, or Lyme disease.

Optical coherence tomography. The OCT (Stratus version 3; OCT 4 Software, Zeiss Meditech, Dublin, California) system was used to obtain circular peripapillary scans (Fast RNFL protocol), which included a set of three $3.4 \mathrm{~mm}$ diameter retinal scans averaged to provide the RNFL thickness at 256 points along the circumference of the circular scan in each eye after mydriasis with $1 \%$ tropicamide. The OCT software employed an automated computerized algorithm to calculate the average thickness of the inner RNFL and to compare these measurements to a normative database of age-matched controls, for patients aged 18 years or older. This algorithm ranked the RNFL measurements against a normal distribution percentile scheme derived from the database of age-matched controls. The mean overall and quadrant (nasal, temporal, superior, and inferior) RNFL measurements obtained for the clinically- affected and clinically - unaffected eyes of patients were assigned either a rank of normal (within the fifth to 95th percentile), above normal (greater than 95th percentile), or below normal (less than fifth percentile) as compared to the database of age-matched controls. A trained ophthalmic medical technologist performed all OCT testing, and monitored scans to ensure that fixation was reliable. Optical coherence tomography scans with a signal strength equal to or greater than 7 (out of 10) were included in the study.

Recurrent Optic Neuritis Events. Patients were monitored for new clinical and sub-clinical ON events in clinically affected and non - affected eyes, which could affect RNFL comparisons between CDMS and non - MS patients. Recurrent clinical ON events were defined as episodes in which patients reported awareness of pain and new onset of vision loss; and had findings consistent with recurrent $\mathrm{ON}$ (worsening visual acuity; a new or worse visual field defect; a new or changed relative afferent pupil defect; and new or worse color vision deficit). Patients who were unaware of a change in their baseline visual function, but who had objective changes on their examination (worsening of visual acuity; a new or worse visual field defect; a new or changed relative afferent pupil defect; and new or worse color vision deficit) to suggest a sub-clinical involvement of the afferent visual pathway were diagnosed with sub-clinical ON.

Statistical analysis. This was a prospective cohort study. Continuous variables were first checked for normality, and then summary statistics were calculated and reported. Median and range were computed for continuous variables that demonstrated a non-Gaussian distribution, including log MAR visual acuity and VFMD; and counts and percentages for categorical variables including conversion to CDMS. For group comparisons, either the Student's t - test or the Kruskal - Wallis rank sum test was used depending on the variable's distribution attribute. Simple logistic regression was performed using conversion to CDMS as the dependent variable and overall RNFL thickness in clinically - affected eyes as the predictor. All statistical analysis was performed using STATA (v. 9, College Station, TX).

\section{Results}

Demographics and Clinical Presentation. Fifty patients (mean age 34 years) were followed for a mean period of 34 months (range 24-44 months). Twenty-one patients (42\%) developed CDMS during the course of the study, with a mean conversion time of 27 months. The baseline demographics and clinical data for all $(n=50)$ patients are included in Table 1 . In the CDMS patient group, 18/21 (86\%) were female; 18/21 (86\%) reported pain at the onset of the ON event; 19/21(91\%) had an abnormal baseline MRI scan; ${ }^{6}$ 14/21 (67\%) had a multi-focal presentation; and 12/21 (57\%) received disease-modifying drug therapy.

The RNFL Values and Future Risk of CDMS. Year one: Mean overall and quadrant RNFL values tended to be thinner in non- affected eyes of non - MS patients; except for the temporal RNFL quadrant, which was thinner in CDMS patients, with a trend towards significance $(p=0.1733)$ (Table 2). In clinically affected eyes, mean RNFL values were significantly thinner in non-MS eyes than CDMS eyes $(\mathrm{p}=0.0462)$. Year two: Differences between RNFL values in non - affected eyes were not significant between non-MS and CDMS patients, albeit temporal RNFL values were again thinner in CDMS patients, with a trend towards significance $(\mathrm{p}=0.1302)$. At this point in follow-up, the relationship between CDMS and non-MS patients reversed, such that RNFL values were thinner in the clinically affected eyes of CDMS patients as compared to non-MS

Table 1: Demographic and clinical characteristics among $\mathrm{ON}$
patients Demographics/Characteristics $(\mathbf{n}=\mathbf{5 0})$

Number of patients 50

Mean age (range)

Male: Female

$\mathrm{CIS}^{*}: \mathrm{CDMS}+$

Pain $(\%)$

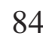

RON $\ddagger$ : AONg

MONO§:MULTI"

$18: 32$

Abnormal MRI\# (\%)

64

Abnormal $\mathrm{CSF}^{* *}(\%)$

70

Steroids^ $(\%)$

50

* Clinically isolated syndrome patients; $†$ Clinically definite MS patients; $¥$ Retrobular optic neuritis patients; IAnterior optic neuritis (or papillitis) patients with mild disc hyperemia at presentation; $\S$ Monofocal ON patients; " Multi-focal ON patients; \# Percentage abnormal magnetic resonance imaging scans at presentation; ${ }^{7} *$ Percentage of patients with positive oligoclonal bands in their cerebrospinal fluid ( $\mathrm{n}=20$ patients): ^ Percentage of patients who received treatment with the equivalent of $1000 \mathrm{mg}$ intravenous methylprednisolone at presentation for optic neuritis. 
Table 2: Comparing RNFL values between CDMS patients and non-MS patients at year one and year two after $\mathrm{ON}$

\begin{tabular}{|c|c|c|c|c|c|c|}
\hline \multirow[b]{2}{*}{$\begin{array}{l}\operatorname{RNFL}^{*} \mathrm{NE}^{\dagger} \\
(\mathrm{CI})^{\#}\end{array}$} & \multicolumn{2}{|c|}{ Year 1} & \multirow[b]{2}{*}{ p-value } & \multicolumn{2}{|c|}{ Year 2} & \multirow[b]{2}{*}{ p-value } \\
\hline & $\begin{array}{l}\text { CDMS }^{\pi} \\
(n=21)\end{array}$ & $\begin{array}{c}\text { Non-MS } \\
(n=29)\end{array}$ & & $\begin{array}{l}\text { CDMS } \\
(n=21)\end{array}$ & $\begin{array}{c}\text { Non-MS } \\
(n=29)\end{array}$ & \\
\hline Overall" & $\begin{array}{c}105.1 \\
(101.5,108.7)\end{array}$ & $\begin{array}{c}102.0 \\
(97.7,106.3)\end{array}$ & 0.2887 & $\begin{array}{c}104.8 \\
(99.2,110.3)\end{array}$ & $\begin{array}{c}103.7 \\
(99.5107 .9)\end{array}$ & 0.7548 \\
\hline Superior & $\begin{array}{c}133.7 \\
(127.2,140.2)\end{array}$ & $\begin{array}{c}130.3 \\
(124.7,136.0)\end{array}$ & 0.4338 & $\begin{array}{c}133.1 \\
(123.4,142.9)\end{array}$ & $\begin{array}{c}135.9 \\
(129.9,141.9)\end{array}$ & 0.6031 \\
\hline Inferior & $\begin{array}{c}134.9 \\
(129.7,140.0)\end{array}$ & $\begin{array}{c}128.1 \\
(121.2,135.0)\end{array}$ & 0.1409 & $\begin{array}{c}132.9 \\
(124.9,140.8)\end{array}$ & $\begin{array}{c}127.2 \\
(121.0,133.4)\end{array}$ & 0.2463 \\
\hline Nasal & $\begin{array}{c}86.6 \\
(79.0,94.2)\end{array}$ & $\begin{array}{c}80.2 \\
(73.6,86.8)\end{array}$ & 0.1995 & $\begin{array}{c}88.1 \\
(79.8,96.5)\end{array}$ & $\begin{array}{c}81.4 \\
(74.0,88.8)\end{array}$ & 0.2216 \\
\hline Temporal & $\begin{array}{c}64.9 \\
(59.3,70.4)\end{array}$ & $\begin{array}{c}69.6 \\
(65.1,74.1)\end{array}$ & 0.1733 & $\begin{array}{c}64.8 \\
(59.4,70.2)\end{array}$ & $\begin{array}{c}70.8 \\
(65.2,76.3)\end{array}$ & 0.1302 \\
\hline $\begin{array}{l}\text { RNFL AE } \\
\text { (CI) }\end{array}$ & $\begin{array}{l}\text { CDMS } \\
(n=21)\end{array}$ & $\begin{array}{c}\text { Non-MS } \\
(n=29)\end{array}$ & & $\begin{array}{l}\text { CDMS } \\
(n=21)\end{array}$ & $\begin{array}{c}\text { Non-MS } \\
(n=29)\end{array}$ & \\
\hline Overall & $\begin{array}{c}89.0 \\
(81.8,96.3)\end{array}$ & $\begin{array}{c}78.3 \\
(71.1,85.9)\end{array}$ & 0.0462 & $\begin{array}{c}81.9 \\
(74.1,89.6)\end{array}$ & $\begin{array}{c}83.1 \\
(75.7,90.6)\end{array}$ & 0.8118 \\
\hline Superior & $\begin{array}{c}115.9 \\
(105.8,126.1)\end{array}$ & $\begin{array}{c}102.3 \\
(91.4,112.4)\end{array}$ & 0.0725 & $\begin{array}{c}105.8 \\
(94.9,116.7)\end{array}$ & $\begin{array}{c}107.0 \\
(97.4,116.6)\end{array}$ & 0.8673 \\
\hline Inferior & $\begin{array}{c}111.3 \\
(101.1,121.5)\end{array}$ & $\begin{array}{c}96.1 \\
(86.2,107.4)\end{array}$ & 0.0464 & $\begin{array}{c}104.0 \\
(91.0,116.9)\end{array}$ & $\begin{array}{c}101.3 \\
(89.0,113.7)\end{array}$ & 0.7694 \\
\hline Nasal & $\begin{array}{c}73.6 \\
(65.5,81.8)\end{array}$ & $\begin{array}{c}63.4 \\
(56.2,72.4)\end{array}$ & 0.0788 & $\begin{array}{c}65.3 \\
(57.8,72.8)\end{array}$ & $\begin{array}{c}70.4 \\
(63.2,77.8)\end{array}$ & 0.3209 \\
\hline Temporal & $\begin{array}{c}55.7 \\
(47.2,64.2)\end{array}$ & $\begin{array}{c}50.9 \\
(45.0,56.2)\end{array}$ & 0.3175 & $\begin{array}{c}52.1 \\
(44.8,59.4)\end{array}$ & $\begin{array}{c}53.5 \\
(47.1,59.9)\end{array}$ & 0.7645 \\
\hline
\end{tabular}

*Retinal nerve fiber layer thickness $(\mu \mathrm{m})$; †Clinically non-affected eyes; $\$$ Clinically affected eyes; IClinically definite multiple sclerosis; § Optic neuritis patients who did not develop CDMS;/ Mean overall RNFL value, and mean RNFL values in the superior, inferior, nasal and temporal quadrants; \# 95\% confidence interval.

patients. Within groups, CDMS patients manifested RNFL thinning in both eyes from year one to year two after ON; whereas RNFL values in non-MS patients marginally increased or stabilized by year two (Table 2).

Logistic regression was performed, using CDMS conversion as the dependent variable, and overall RNFL value in clinically affected eyes to predict the odds of conversion to CDMS after ON. The simple logistics regression model yielded an odds ratio of $1.033(\mathrm{p}=0.0427)$ for year one and 0.9962 for year two $(p=0.8071)$. These results indicate that overall RNFL thickness had limited use in predicting CDMS conversion one year after $\mathrm{ON}$, and its predictive value diminished thereafter. Given that all other clinical conditions remained unchanged, a $5 \mu \mathrm{m}$ increase in mean the RNFL thickness of a clinically - affected eye increased the odds of converting to CDMS 1.18 fold one year after ON. Otherwise stated, for every $1 \mu \mathrm{m}$ increase in RNFL thickness in clinically - affected eyes, ON patients increased their chance of converting to CDMS at one year by $3.3 \%$.

Visual recovery after $O N$ : Visual field mean deviation and $\log$ MAR visual acuity scores were compared between the two groups at year one and year two after ON. Non-MS patients manifested less complete visual recovery than CDMS patients in this study. More specifically, six non-MS patients and two CDMS patients had visual acuity scores worse than 20/40 in AE; and seven non-MS patients and two CDMS patients manifested VFMD scores worse than $-5.00 \mathrm{~dB}$ in clinically - affected eyes after ON. Median log MAR visual acuity and VFMD scores were worse in both eyes of non-MS patients as compared to CDMS patients at year one and year two after ON, albeit differences between the two groups were not significant (Table 3 ).

Recurrent ON events: Recurrent ON events occurred more frequently in CDMS patients than non-MS patients. Sub-clinical ON Events: Five patients (four CDMS patients and one non - MS patient) developed progressive RNFL thinning ( $>10 \mu \mathrm{m})$ and associated changes in visual function in the original non affected eye during year two of the study. These patients did not report awareness of pain or vision loss with their sub-clinical ON events. Three CDMS patients developed objective worsening of visual function scores and RNFL thinning during the second year of the study, consistent with recurrent sub-clinical ON in the original clinically - affected eye. Clinical ON Events: Two CDMS patients underwent assessment for recurrent clinical ON in their original non - affected eye in year two of the study. In both cases progressive RNFL thinning $(>10 \mu \mathrm{m})$ was observed. 
Table 3: Visual outcomes in CDMS patients and non- MS patients after ON

\begin{tabular}{|c|c|c|c|c|c|c|}
\hline \multirow[b]{2}{*}{ Year 1} & \multicolumn{3}{|c|}{$\mathrm{AE}^{*}$} & \multicolumn{3}{|c|}{$\mathbf{N E}^{\dagger}$} \\
\hline & CDMS $^{\ddagger}$ & Non-MS ${ }^{\text {II }}$ & p-value & CDMS & Non-MS & p-value \\
\hline $\log$ MAR VA ${ }^{\S}$ & 0.00 & 0.05 & 0.706 & 0.00 & 0.00 & 0.674 \\
\hline VFMD & -1.14 & -2.04 & 0.556 & -0.38 & -0.70 & 0.239 \\
\hline Year 2 & & & & & & \\
\hline log MAR VA & 0.00 & 0.05 & 0.614 & 0.00 & 0.00 & 0.714 \\
\hline VFMD & -1.29 & -3.05 & 0.732 & -0.79 & -0.91 & 0.843 \\
\hline
\end{tabular}

*Clinically affected eyes; $\dagger$ clinically unaffected eyes; $\$$ clinically definite MS; 9 optic neuritis patients who did not develop CDMS: § median log MAR visual acuity; II median visual field mean deviation.

Two CDMS patients experienced recurrent clinical ON in their original clinically - affected eye during the second year of follow-up. One patient manifested progressive RNFL thinning (> $10 \mu \mathrm{m})$, whereas the second patient maintained stable RNFL values in the clinically - affected eye.

\section{DISCUSSION}

In this study, we demonstrated that RNFL values differ between CDMS and non-MS patients after ON. After one year, RNFL values were significantly thinner in the clinically affected eyes of non-MS patients, which may have been due to less complete visual recovery from $\mathrm{ON}$ in this group. In the second year of follow-up, CDMS patients manifested more recurrent $\mathrm{ON}$ events, and demonstrated greater thinning of the RNFL than non-MS patients. CDMS patients had greater atrophy in the temporal RNFL region in non - affected eyes than non MS patients, with a trend toward significance.

Prior studies have shown that RNFL values are reduced in MS patients as compared to healthy control subjects, with the lowest RNFL values reported in eyes previously affected by ON. ${ }^{11,18}$ Henderson and colleagues ${ }^{19}$ recently compared RNFL thickness and macular volumes in 23 patients with primary progressive MS, 27 patients with secondary progressive MS, and 20 healthy controls. While their results indicated significant reductions in RNFL thickness and macular volume in the eyes of secondary progressive MS patients not previously affected by $\mathrm{ON}$, the authors concluded that "clinically overt ON has adverse consequences for the RNFL over and above that of secondary progressive MS alone." There have been no prior studies, which have tracked RNFL changes after ON to determine the future risk of CDMS. Our results are consistent with related reports, and indicate that lower RNFL values manifest in patients with less complete recovery after ON, irrespective of MS status. The findings from our study confirm that RNFL thinning is heavily dependent on the severity of the $\mathrm{ON}$ event; and that as a surrogate biomarker for axonal integrity, RNFL thickness has limited use in predicting CDMS conversion in first year of follow up after ON.

We observed that differences in RNFL thickness were not significant between CDMS and non-MS patients two years after $\mathrm{ON}$; but progressive RNFL thinning was more apparent in the clinically - affected eyes of CDMS patients than non-MS patients during the second year of this study. Accordingly, recurrent subclinical ON events occurred with greater frequency in CDMS patients, which correlated to the progressive RNFL thinning observed in this group. This observation suggests that subclinical insults to the afferent visual pathway cause progressive axonal attrition, which is the substrate for reduced RNFL values in MS patients, with and without a history of ON. ${ }^{11,18}$ Our results indicate that cross-sectional comparisons do not aptly capture disease dynamics, which distinguish patients at greater risk for developing CDMS after ON. Instead, longitudinal studies designed to track RNFL changes and detect recurrent ON events may better identify patients at risk for future MS. More specifically, OCT may have a role in detecting ON patients at risk for CDMS, by revealing trends of change in RNFL thickness over time, which mirror sub-clinical disease activity in the afferent visual pathway.

There were limitations in this study, which likely impacted our results. First, the follow up period of two years may not have been an adequate time interval to detect significant differences in RNFL thickness, which distinguish CDMS from non-MS patients. Secondly, the small size of our patient population hindered the statistical power of comparisons between RNFL values in CDMS and non-MS patients. We observed that temporal RNFL values in non - affected ON eyes were generally thinner in CDMS than non-MS patients; with a trend towards significance. It is possible that in a larger study population, a more significant relationship between temporal RNFL thinning and future CDMS risk would have emerged. In a recent twoyear prospective study, Sepulcre et al showed that temporal quadrant RNFL values were reduced in MS patients; and that baseline temporal RNFL atrophy was associated with the presence of new relapses and MS-related disability. ${ }^{16}$ Furthermore, Henderson and colleagues ${ }^{19}$ reported that RNFL loss was most evident in the temporal quadrant of progressive MS patients; such that significant reduction was seen in primary progressive MS versus controls and in secondary versus primary progressive MS. Therefore, it is plausible that temporal RNFL atrophy may distinguish patients at increased risk for CDMS after ON, but this remains to be established. 
In conclusion, we documented RNFL changes and events in $\mathrm{ON}$ patients over a two-year period to aid in the design of future intervention studies. Our results indicate that RNFL values should be followed longitudinally in concert with tests of visual function to fully capture the effects of sub-clinical disease activity on structural RNFL measures in the afferent visual pathway. Trends of change in RNFL thickness, and temporal atrophy may be used to identify patients at increased risk for CDMS after ON, but this awaits further study. Future prospective clinical trials should include a larger patient population than we used in this study, to determine whether RNFL changes after ON may be used to identify patients at risk for future CDMS.

\section{ACKNOWLEGEMENTS}

This study was supported by a grant received (FC) from the E.A. Baker Foundation, the Canadian National Institute for the Blind.

\section{REFERENCES}

1. Hickman SJ, Dalton CM, Miller DH, Plant GT. Management of acute optic neuritis. Lancet. 2002; 360 (9349): 1953-62.

2. Miller D, Barkhof F, Montalban X, Thompson A, Filippi M. Clinically isolated syndromes suggestive of multiple sclerosis, part I: natural history, pathogenesis, diagnosis, and prognosis. Lancet Neurol. 2005; 4 (5): 281-8.

3. Beck RW, Cleary PA, Anderson MM Jr, Keltner JL, Shults WT, Kaufman DI, et al. Optic Neuritis Study Group. A randomized, controlled trial of corticosteroids in the treatment of acute optic neuritis. N Engl J Med. 1992; 326 (9): 581-8.

4. Optic Neuritis Study Group. The 5-year risk of MS after optic neuritis. Experience of the Optic Neuritis Treatment Trial. Neurology. 1997; 49 (5): 1404-13.

5. Beck RW, Trobe JD, Moke PS, Gal RL, Xing D, Bhatti MT, et al. High- and low-risk profiles for the development of multiple sclerosis within 10 years after optic neuritis: experience of the Optic Neuritis Treatment Trial. Arch Ophthalmol. 2003; 121 (7): 944-9.

6. Jacobs LD, Beck RW, Simon JH, Kinkel RP, Brownscheidle CM, Murray $\mathrm{TJ}$, et al. Intramuscular interferon beta-1a therapy initiated during a first demyelinating event in multiple sclerosis. CHAMPS Study Group. N Engl J Med. 2000; 343 (13): 898-904.

7. Comi G, Filippi M, Barkhof F, Durelli L, Edan G, Fernandez O, et al. Effect of early interferon treatment on conversion to definite multiple sclerosis: a randomised study. Lancet. 2001; 357 (9268): 1576-82.

8. Kappos L, Polman CH, Freedman MS, Edan G, Hartung HP, Miller $\mathrm{DH}$, et al. Treatment with interferon beta- $1 \mathrm{~b}$ delays conversion to clinically definite and McDonald MS in patients with clinically isolated syndromes. Neurology. 2006; 67(7): 1242-9.

9. Parisi V, Manni G, Spadaro M, Colacino G, Restuccia R, Marchi S, et al. Correlation between morphological and functional retinal impairment in multiple sclerosis patients. Invest Ophthalmol Vis Sci. 1999; 40 (11): 2520-7.

10. Trip SA, Schlottmann PG, Jones SJ, Altmann DR, Garway - Heath DF, Thompson AJ, et al. Retinal nerve fiber layer axonal loss and visual dysfunction in optic neuritis. Ann Neurol. 2005; 58 (3): 383-91.
11. Fisher JB, Jacobs DA, Markowitz CE, Galetta SL, Volpe NJ, Nano - Schiavi ML, et al. Relation of visual function to retinal nerve fiber layer thickness in multiple sclerosis. Ophthalmology. 2006; 113 (2): 324-32.

12. Noval S, Contreras I, Rebolleda G, Munoz-Negrete FJ. Optical coherence tomography versus automated perimetry for followup of optic neuritis. Acta Ophthalmol Scand. 2006; 84 (6): 790-4.

13. Costello F, Coupland S, Hodge W, Lorello GR, Koroluk J, Pan YI, et al. Quantifying axonal loss after optic neuritis with optical coherence tomography. Ann Neurol. 2006; 59 (6): 963-9.

14. Trip SA, Schlottmann PG, Jones SJ, Li WY, Garway - Heath DF, Thompson AJ, et al. Optic nerve atrophy and retinal nerve fiber layer thinning following optic neuritis: evidence that axonal loss is a substrate of MRI-detected atrophy. Neuroimage. 2006; 31 (1): 286-93.

15. Trip SA, Schlottmann PG, Jones SJ, Li WY, Garway - Heath DF, Thompson AJ, et al. Optic nerve magnetization transfer imaging and measures of axonal loss and demyelination in optic neuritis. Mult Scler. 2007; (7): 875-9.

16. Sepulcre J, Murie - Fernandez M, Salinas - Alaman A, Garcia Layana A, Bejarano B, Villoslada P. Diagnostic accuracy of retinal abnormalities in predicting disease activity in MS. Neurology. 2007; 68 (18): 1488-94.

17. Gordon - Lipkin E, Chodkowski B, Reich DS, Smith SA, Pulicken $\mathrm{M}$, Balcer LJ, et al. Retinal nerve fiber layer associated with brain atrophy in multiple sclerosis. Neurology. 2007; 69 (16): 1603-9.

18. Pulicken M, Gordon - Lipkin E, Balcer LJ, Frohman E, Cutter G, Calabresi PA, et al. Optical coherence tomography and disease subtype in multiple sclerosis. Neurology. 2007; 69 (22): 2085-92.

19. Henderson AP, Trip SA, Schlottmann PG, Altmann DR, Garway Heath DF, Plant GT, et al. An investigation of the retinal nerve fiber layer in progressive multiple sclerosis using optical coherence tomography. Brain. 2008; 131: 277-87.

20. Huang D, Swanson EA, Lin CP, Schuman JS, Stinson WG, Chang W, et al. Optical coherence tomography. Science. 1991; 254 (5035):1178-81.

21. Baumal CR. Clinical applications of optical coherence tomography. Curr Opin Ophthalmol. 1999; 10 (3): 182-8.

22. Paunescu LA, Schuman JS, Price LL, Stark PC, Beaton S, Ishikawa $\mathrm{H}$, et al. Reproducibility of nerve fiber thickness, macular thickness and optic nerve head measurements using Stratus OCT. Invest Ophthalmol Vis Sci. 2004; 45 (6):1716-24.

23. Schuman JS, Hee MR, Puliafito CA, Wong C, Pedut - Kloizman T, Lin CP, et al. Quantification of nerve fiber layer thickness in normal and glaucomatous eyes using optical coherence tomography a pilot study. Arch Ophthalmol. 1995;113 (5): 586-96.

24. Frisen LF, Hoyt WF. Insidious atrophy of retinal nerve fibers in multiple sclerosis. Arch Ophthalmol. 1974; 92 (2):91-7.

25. Poser CM, Paty DW, Sheinberg L, McDonald WI, Davis FA, Ebers $\mathrm{GC}$, et al. New diagnostic criteria for multiple sclerosis: guidelines for research protocols. Ann Neurol. 1983; 13 (3): 227-31.

26. Polman CH, Reingold SC, Edan G, Filippi M, Hartung HP, Kappos L, et al. Diagnostic criteria for Multiple Sclerosis: 2005 revisions to the "McDonald Criteria". Ann Neurol. 2005; 58 (6): 840-6.

27. Ferris FL III, Kassoff A, Bresnick GH, Bailey L. New visual acuity charts for clinical research. Am J Ophthalmol. 1982; 94 (1): 91-6. 